\title{
MEROMORPHIC FUNCTIONS AND SMOOTH ANALYTIC FUNCTIONS
}

\author{
ROBERT KAUFMAN
}

\begin{abstract}
Meromorphic functions with many zeroes can have logarithmic derivatives that are relatively smooth. We prove this, with a new construction of smooth analytic functions with many zeroes. Our examples belong to the theory of differential fields of functions.
\end{abstract}

In this note we consider functions $y$, meromorphic in the disk $|z|<1$, and their logarithmic derivatives $L(y)=y^{\prime} / y$. Plainly, zeroes and poles of $y$ are poles of $L(y)$, but the multiplicity of the zeroes and poles is not easily controlled. When $L(y)$ is a function of bounded characteristic, i.e. a quotient of bounded analytic functions in $|z|<1$, the sequence $S=\left(z_{k}\right)_{1}^{\infty}$ of zeroes of $y$ must fulfill the Blaschke condition $\Sigma 1-\left|z_{k}\right|<+\infty$, but S. Bank proved recently [1] that the multiplicities of the zeroes $z_{k}$ can be determined arbitrarily, if only $\Sigma 1-\left|z_{k}\right|<+\infty$.

The set of functions of bounded characteristic forms a field, but not a differential field; indeed, W. Rudin [4] constructed a bounded analytic function $g$ such that $\int_{0}^{1}\left|g^{\prime}\left(r e^{i \theta}\right)\right| d r=+\infty$ for almost all $\theta$, whence $g^{\prime}$ is not of bounded characteristic. (See also [5].) Let now $A^{\infty}$ be the class of functions $g$, such that each derivative $g^{(n)}$ is bounded in $|z|<1$, and $M^{\infty}$ the field of quotients of $A^{\infty}$. Clearly $M^{\infty}$ is a differential field of functions.

THEOREM. Let $g \in A^{\infty}, g \not \equiv 0$, and let $S=\left(z_{k}\right)_{1}^{\infty}$ be the zero-set of $g$ in $|z|<1$. Then for any sequence $\left(n_{k}\right)$ of nonnegative integers, there is a meromorphic function $y$, with zeroes at $z_{k}$ of multiplicity $n_{k}$ (and no other zeroes) such that $L(y)$ is in $M^{\infty}$.

In the proof of our theorem we need a precise description of possible zero-sets $S$, obtained in [3] and [7]. In an Appendix we derive this description by a method rather different from [3], [7].

It is easy to derive a necessary property of $S$ in terms of the function $\rho(z)=\inf \{|z-s|: s \in S\}$, since $\left|g\left(e^{i \theta}\right)\right| \leqslant C \rho\left(e^{i \theta}\right)$. Now it is clear that $\log \rho\left(e^{i \theta}\right)$ must be integrable on $(0,2 \pi)$; in combination with the Blaschke condition, this is sufficient.

Received by the editors April 22, 1976.

AMS (MOS) subject classifications (1970). Primary 30A76, 30A78; Secondary 46E15, 30A70.

Key words and phrases. Logarithmic derivative, conformal mapping, Poisson integral, zero set. 
Let now $S=\left(z_{k}\right)$ be a zero-set as in the statement of the theorem. We choose $z_{k}^{*} \notin S$ so that

$$
2\left|z_{k}^{*}-z_{k}\right|<1-\left|z_{k}\right| \text { and } n_{k}\left|z_{k}-z_{k}^{*}\right| \leqslant C_{r}\left(1-\left|z_{k}\right|\right)^{r+2} k^{-2}
$$

for $r=1,2,3, \ldots$ We set $S_{1}=\left(z_{k}\right) \cup\left(z_{k}^{*}\right)$, and observe $S_{1}$ is a Blaschke sequence, while $\left|e^{i \theta}-z_{k}\right| \leqslant 2\left|e^{i \theta}-z_{k}^{*}\right|$, so thatat $S_{1}$ is the zero-set of some function $g_{1}$ in $A^{\infty}$.

We assert now that the series

$$
h_{N}=g_{1} \sum_{1}^{N} n_{k}\left[\left(z-z_{k}\right)^{-1}-\left(z-z_{k}^{*}\right)^{-1}\right]
$$

converges uniformly on $|z|<1$, together with all of its derivatives. To verify this, we have only to estimate the derivatives on the boundary $|z|=1$, and by Leibniz' formula we can omit the factor $g_{1}$. The $r$ th derivative is then

$$
\sum_{1}^{N}(-1)^{r} r ! n_{k}\left[\left(z-z_{k}\right)^{-1-r}-\left(z-z_{k}^{*}\right)^{-1-r}\right] \text {. }
$$

Now

$$
(\partial / \partial w)(z-w)^{-1-r}=(1+r)(z-w)^{-2-r},
$$

so that

$$
\left|\left(z-z_{k}\right)^{-1-r}-\left(z-z_{k}^{*}\right)^{-1-r}\right| \leqslant C_{r}^{\prime}\left|z_{k}-z_{k}^{*}\right|\left(1-\left|z_{k}\right|\right)^{-2-r}
$$

on the boundary $|z|=1$. We find that $\lim h_{N}=h$ belongs to $A^{\infty}$, and for the function $y=\Pi\left(z-z_{k}\right)^{n_{k}}\left(z-z_{k}^{*}\right)^{-n_{k}}$ we have $L(y)=h g_{1}^{-1}$ in $M^{\infty}$.

Appendix. Let $S$ be a Blaschke sequence such that $\log \rho\left(e^{i \theta}\right)$ is integrable. There is a function $\delta\left(e^{i \theta}\right)$, of class $C^{2}$ on $|z|=1$, such that $C_{1} \rho^{2}\left(e^{i \theta}\right) \leqslant \delta\left(e^{i \theta}\right)$ $\leqslant \rho^{2}\left(e^{i \theta}\right)$. In fact $\delta$ is nothing but the square of the "regularized distance" $\Delta$ to the set $S\left[6\right.$, p. 171]. Let now $D^{+}$be the region bounded by the curve $r=1+\delta\left(e^{i \theta}\right)$, so $D^{+} \supseteq D$ and $D^{+}$is a region of class $C^{2}$. There exists a conformal mapping $\Phi$ of $D^{+}$onto $D$ such that $\Phi$ and $\Phi^{-1}$ both have derivatives continuous up to the boundary, and even Hölder-continuous [2, p.374]; whence $a\left|z_{1}-z_{2}\right| \leqslant\left|\Phi\left(z_{1}\right)-\Phi\left(z_{2}\right)\right| \leqslant b\left|z_{1}-z_{2}\right|$ for certain constants $a>0, b>0$ (Kellogg's theorem). The distance of $z_{k}$ from $\partial D^{+}$is at most $1-\left|z_{k}\right|+\delta\left(e^{i \theta}\right)$ if $z_{k}=e^{i \theta}\left|z_{k}\right|$, so the distance is at most $2\left(1-\left|z_{k}\right|\right)$. Consequently $1-\left|\Phi\left(z_{k}\right)\right|=O\left(1-\left|z_{k}\right|\right)$, whence $\Phi(S)$ is a Blaschke sequence in $D$, and there is a bounded analytic function $B$, on $D^{+}$, with zero-set $S$.

Let $\mu_{z}$ be the harmonic measure on $\partial D^{+}$for the point $z$. By the differentiability properties of $\Phi$ and $\Phi^{-1}$, we see that $L^{1}(d \theta)$ and $L^{1}\left(d \mu_{0}\right)$ can be identified. Moreover, elementary geometry yields the inequality $\rho\left(\operatorname{Re}^{i \theta}\right)>$ $\rho\left(e^{i \theta}\right)$ when $R>1$, so that $\log \rho(w)$ belongs to $L^{1}\left(d \mu_{0}\right)$. By a classical method we can find a monotone function $\psi(t)$ on $t>0$, such that $\psi(t) \geqslant t+1$ and $\psi(t) / t \rightarrow+\infty$ as $t \rightarrow+\infty$ and $\psi(|\log \rho(w)|)$ is in $L^{1}\left(d \mu_{0}\right)$. Let $u$ be its Poisson integral on $D^{+}, v$ the harmonic conjugate of $u$, and $g=e^{-u-i v} B$. 
Before completing the proof that $g$ belongs to $A^{\infty}$, we observe an inequality on $\mu_{z}$ for $z$ near $\partial D^{+}$. Let $\Gamma_{z}$ be the part of the boundary defined by the inequality $|z-w|<3 d\left(z, \partial D^{+}\right)$. Using the continuity of $\Phi^{\prime}$ we find that $\mu_{z}\left(\Gamma_{z}\right) \geqslant a>0$ for all $z$ in $D^{+}$. On $\Gamma_{z}$ we have $\rho(w) \leqslant \rho(z)+3 d\left(z, \partial D^{+}\right)$, so that, if $\rho(z)+d\left(z, \partial D^{+}\right)$is small, $u(z)$ is a large multiple of

$$
-\log \left[\rho(z)+d\left(z, \partial D^{+}\right)\right]
$$

and $|g(z)|$ is bounded by a large power of $\rho+d$. Thus, for each $N>0$

$$
|g(z)| \leqslant C_{N}\left[\rho(z)+d\left(z, \partial D^{+}\right)\right]^{N} \text {. }
$$

Around each $z$ in $D^{+}$we draw a disk of radius $d\left(z, \partial D^{+}\right) / 2$, and observe that the bound for $g$ is increased by at most $2^{N}$. Cauchy's formulas give, for $r=0,1,2, \ldots$,

$$
\left|g^{(r)}(z)\right| \leqslant C_{N, r}\left[\rho(z)+d\left(z, \partial D^{+}\right)\right]^{N} / d^{r}(z, \partial D) .
$$

Let $\varepsilon>0$, and observe that on the set defined by the inequality $d\left(z, \partial D^{+}\right) \geqslant$ $\varepsilon \rho^{2}(z)$, each $g^{(r)}$ is uniformly bounded. But for small $\varepsilon>0$, this set contains the disk $|z|<1$. Indeed $d\left(|z| e^{i \theta}, \partial D^{+}\right) \geqslant 1-|z|+\varepsilon^{\prime} \rho^{2}\left(e^{i \theta}\right)$, so the inequality is true as soon as $\varepsilon^{\prime}(1-2 \varepsilon)^{2} \geqslant \varepsilon$.

In the proof just completed, $S$ could have contained points on $\partial D$, in which case all the derivatives $g^{(r)}$ vanish on $S \cap \partial D$. It is also worth remarking that a Blaschke sequence contained in a ball $|z-r| \leqslant 1-r(0<r<1)$ is a zero-set for $A^{\infty}$, because $\rho\left(e^{i \theta}\right) \geqslant\left|e^{i \theta}-r\right|-1+r>a \theta^{2}$ for small $\theta$.

\section{REFERENCES}

1. S. Bank, On the existence of meromorphic solutions of differential equations having arbitrarily rapid growth, J. Reine Angew. Math. (to appear).

2. G. M. Golusin, Geometrische Funktionentheorie, VEB Deutscher Verlag, Berlin, 1957. MR $19,735$.

3. J. D. Nelson, $A$ characterization of zero sets for $A^{\infty}$, Michigan Math. J. 18 (1971), 141-147. MR $44 \# 410$.

4. W. Rudin, The radial variation of analytic functions, Duke Math. J. 22 (1955), 235-242. MR 18, 27.

5. On a problem of Bloch and Nevanlinna, Proc. Amer. Math. Soc. 6 (1955), 202-204. MR 16, 810.

6. E. M. Stein, Singular integrals and differentiability properties of functions, Princeton Univ. Press, Princeton, N.J., 1970. MR 44 \#280.

7. B. A. Taylor and D. L. Williams, Zeros of Lipschitz functions analytic in the unit disc, Michigan Math. J. 18 (1971), 129-139. MR 44 \# 409.

Department of Mathematics, University of Illinois at Urbana-Champaign, Urbana, ILLINOIS 61801

Current address: Swain Hall East, Indiana University, Bloomington, Indiana 47401 